endothelioma, attached to the upper and inner wall of the orbit, which had perforated the bone, a swelling of the frontal bone was noticed, particularly of the outer portion, while the temporal bone also showed enlargement. The condition was treated by $\mathrm{x}$-rays, in full therapeutic doses, for five minutes once a month, which succeeded in holding the process in check for several years, while, as shown by $\mathrm{x}$-ray plates, regeneration of bone took place in the frontal region. The man, however, died with cerebral manifestations ten years after the first symptons of the orbital growth had been observed.

\title{
CORRESPONDENCE
}

\section{FACTORS IN STEREOSCOPIC VISION}

To the Editor of THE British Journal of Ophthalmology.

SIR,-I have read with great pleasure the interesting contribution of Dr. E. M. Eaton on "Factors in Stereoscopic Vision and the Visual Estimation of Distance," which appeared in the February number of the Journal. On repeating, however, the experiments with the stereoscope which he describes, I find I am unable to agree with his statements of fact, and consequently with the deductions therefrom.

If an ordinary stereoscopic photographic view be divided into two halves and the left half, i.e., that part of the picture which is placed in the stereoscope opposite the left eye, be placed in the stereoscope opposite my left eye, I see it double. The view seen by the left eye is projected well towards the right, while the portion of the picture seen by the right eye is projected towards the position which the picture actually occupies. With the right half of the picture before the right eye, duplication occurs again, the projection being as already described. The two views in each case show distance and solidity very clearly indeed, the erroneously projected view being lighter than the other. If now the left half of the picture be placed in the stereoscope before the left eye and the right half of the picture brought forward so that the projected view of the left is overlapped by the correctly placed view of the right eye, the resultant view shows depth and solidity well marked. By reversing the pictures, the only difference I found between the perspective views due to one eye and that due to the use of two, was that the combined view was somewhat blurred in its fine details. 
If we invert the right half and place it in that position before the left eye in the stereoscope, it is seen double again; but in this case the sense of perspective is faulty. In some cases there is no difficulty in interpreting the view for it can be compared to the reflection of hills in the still waters of a lake. In other cases another interpretation is possible, as in the "six are seven cubes" illusion; we interpret the shadows of the various objects in a wholly different manner, and an absolutely new picture starts into view. Not all of the old view appears in the new. Which of the two views will predominate when the second half of the original picture is inverted and approximated to that in the stereoscope will depend on circumstances. If, as already stated, the inverted right half is placed in the stereoscope before the left eye and the inverted left half is brought into its proper position before the right eye, the view obtained is the water view. With the inverted left half before the left eye and the inverted right approximated thereto in the stereoscope the alternative view becomes prominent, depth and solidity being very pronounced. The parts of the original picture which are not essential to the new view apparently come forward, causing a relative movement of the new view backward. The parts which move forward may be bits from the fore or background; in some cases they appear like a painted screen between the observer and the distant view and in some, if not in all, cases can be blocked out without altering the new view.

According to this rendering of the facts, the foreground is not thrown into the distance and distant objects brought forward when the inverted and reversed halves of a stereoscopic picture are seen in a stereoscope; but a new view is seen in which the fusion sense has full play in the ordinary acceptation of the term.

As a consequence, the experiment does not support the contention that "Our fusion sense appears to be extremely elastic and readily capable of compromise or even suppression."

$$
\begin{gathered}
\text { Yours, etc., } \\
\text { T. Stewart Barrie, M.B., F.R.F.P.S.G. }
\end{gathered}
$$

13, Somerset Place,

GLASGOW, W.

20 February, 1919

\section{RING INFILTRATION OF THE CORNEA IN PLAGUE}

\section{To the Editor of The British Journal of Ophthalmology.}

SIR,-In the December, 1918, issue of the BRITISH Journal OF OPHTHALMOLOGY, page 612, Mr. D. V. Giri states that no case of acute general infection has been reported as a cause of ring 